\title{
ENTREVISTA A EUGENIO COSERIU
}

\author{
Ricardo Mairal \\ Universidad Autónoma de Madrid \\ Pedro Santana \\ Colegio Universitario de La Rioja
}

El texto que ofrecemos a continuación es el resultado de la transcripción de parte de una entrevista mantenida con el gran lingüista rumano, la cual tuvimos ocasión de realizar con motivo de su participación en el curso El componente léxico. Enfoques funcionales y formales, organizado por la Universidad de Granada y que tuvo lugar en Almuñécar en septiembre de 1990.

El lector podrá comprobar cómo el insigne lingüista va repasando alguno de los temas centrales de su reflexión. En ese sentido, hay que decir que, debido a la exhaustividad y precisión de sus respuestas, la arquitectura de la entrevista es más obra suya que nuestra y creemos que este hecho la hace más interesante.

Debemos expresar nuestro más profundo agradecimiento al Profesor Coseriu por su extraordinaria generosidad y por la amabilidad que tuvo al acceder a esta entrevista. También es de justicia expresar aquí el agradecimiento que debemos al Profesor Martín Mingorance, director del curso, quien nos facilitó los medios y las circunstancias que permitieron nuestro trabajo y al Profesor García Turza, cuyos certeros comentarios ayudaron a aclarar algún extremo de la transcripción.

-Para comenzar, ¿podría hablarnos acerca de su formación como lingüista? $i$ de qué maestros recibió una mayor influencia? ¿cuál era el ambiente cultural de la Europa en que usted se formó?

- En cierto sentido he tenido varios maestros y, en otro sentido, prácticamente no he tenido maestros propiamente dichos. En la edición española de las Lecciones de Lingüística General he puesto un prefacio y además dedico el libro a la memoria de tres grandes maestros, que fueron Giovanni Maver, profesor de Eslavistica en Roma, Antonino Pagliaro, profesor de Lingüística también en Roma y Antonio Banfi, profesor de Filosofía en Milán. Pero en ese prefacio especifico que no es que haya 
recibido de ellos la información, sino la formación intelectual $\mathrm{y}$, digamos, la educación crítica abierta y el considerar cualquier tipo de teoría y de método en lo posible con simpatía y desde dentro. Tratar de entender cada uno de los métodos y cada uno de los planteamientos por sus mismas razones, en lugar de considerarlos desde fuera, desde una posición dogmática; lo que yo resumo en una sola palabra : que lo que yo he aprendido de ellos es el anti-dogmatismo.Sin embargo, no puedo decir que me hayan formado como lingüista porque el profesor Maver era profesor de Filología Eslava, en realidad. Me interesaba más su actitud frente a los proble mas. A los cursos de Pagliaro casi no asistí y fue maestro mío más bien a través de los escritos, mientras que Maver sí dirigió mi primera tesis doctoral y Banfi mi segunda tesis doctoral en filosofía; pero yo no había asistido nunca a ninguna clase de Banfi. Así que no es que me hayan formado en lo que concierne a la información, o a un método determinado, a un enfoque determinado.

Yo puse en una pequeña biografía que se nos pidió para Trends in Linguistics que mis maestros en relación con la lingüistica habian sido Aristóteles, Hegel y Humboldt. Aparte, he de decir que precisamente no he tenido maestros pues no continúo ningún tipo de escuela, sino que como lingüista me he formado solo, desarrollando en realidad la teoría lingüística sobre una única base, la cual es el principio de toda ciencia: el decir las cosas como son, que es lo más difícil y no sé si será necesario explicar esto más de cerca. Pueden leer la Introducción a mi libro Gramática, Semántica, Universales . Allí he puesto cuáles son los principios. Primero, decir las cosas como son, que es un principio enunciado por Platón en El Sofista , que dice precisamente esto, que el $\lambda$ ójo $\varsigma$ verdadero dice las cosas como son; mientras que el $\lambda$ ójoৎ falso dice las cosas como no son, o como han dejado de ser o como no son todavia. Esto en las ciencias humanas significa primero que en realidad se trata de trasladar al plano de la reflexividad aquello que también todo hablante sabe como hablante, porque el fundamento de las ciencias humanas, no en lo particular, sino en lo universal, es el saber originario que el hombre tiene acerca de sí mismo y de sus propias actividades. El hombre, el hablante, sabe lo que es el lenguaje, sabe lo que es la lengua, sabe lo que es el significado, etc. Aquí se trata sólo de trasladar este saber, y éste es el cometido de la teoria, desde el plano del saber intuitivo al plano del saber justificado. En realidad es esto lo que hace el lingüista en tanto teórico de la lengua.

Tenemos a veces la impresión de que el lingüista dice cosas que todos sabemos y esto es cierto. Tiene que decir cosas que todos sabemos en cuanto hablantes; e incluso cosas que él mismo sabe como hablante, sólo que el hablante lo sabe sin ningún tipo de justificación. Lo sabe haciéndolo. Lo mismo hablando que entendiendo, mientras que el lingüista explica y trata de ver cuál es el fundamento en cada caso. El cometido de una ciencia humana en general, en la teoría, es trasladar lo intuitivo al plano de la reflexividad, o sea, transformar el saber que Leibniz llama saber claro-oscuro en saber distinto y adecuado. Como dice Hegel, se trata aqui de trasladar lo que es gekannt, conocido, sabido, a lo que es erkannt, es decir, conocimiento fundado. Y esto entonces indica otra cosa todavia: que puesto que se trata de aquello que todos saben, esto implica que también es sabido todavía y por tanto que en la tradición se encontrará no sólo atisbos, sino ideas y desarrollos importantes y que el lingüista, 
como cualquier otro científico en el campo de lo humano, tiene que referirse necesariamente a la tradición, por respeto a estos otros hombres que han pensado lo mismo y que han tratado de decir lo mismo.

Lo que ocurre es que es muy difícil decir las cosas como son y siempre se ven en una sola perspectiva; y eso es lo que tenemos que hacer en la interpretación de la tradición: interpretación positiva, ver que efectivamente cada uno de los que con seriedad y sinceridad se han dedicado a la teoría del lenguaje han tenido que ver algo. Quizá algo desviado, parcializado, etc., pero hay que encontrar este núcleo positivo, que yo resumo en dos fórmulas: que ningún error es pura y simplemente error, sino que debe contener algo de verdad, o alguna motivación. Además, segundo, en las ciencias humanas tenemos siempre la tradición y, dentro de la tradición, lo nuevo, lo novedoso, incluso lo revolucionario. Por lo cual, quien sólo dice algo nuevo no dice nada nuevo. Y, finalmente, como pricipio deducido de esto, si se trata de la tradición, ha de ser el caso que otros lingüistas actuales tengan sus razones. Eso significa también que esta ciencia la hacemos para los hablantes mismos, para los usuarios; significa que el hablante tiene siempre razón en cuanto hablante y puede no tenerla en cuanto lingüista, cuando habla del lenguaje. Cuando está en el lenguaje y habla o entiende, siempre tiene razón, porque el lenguaje - y ésta es otra de mis fórmulas- funciona por y para los hablantes y no por y para los lingüistas. Esto significa también que nosotros necesitamos el saber intuitivo del hablante y que después tenemos que explicárselo de una manera cultural y socialmente útil, lo cual quiere decir, sin excesivo formalismo, sin excesiva formalización.

La formalización se da para otros aspectos, no para la teoría. Para el trabajo técnico, pero no para la teoría lingüística. El lingüista también ha de utilizar, en la medida de lo posible, aquellos conceptos que han acuñado con respecto al lenguaje las lenguas mismas. De aquí que yo tienda a trasladar al plano de la terminología, de los términos definidos, los conceptos usuales como designar, nombrar, significado, sentido, etc.

- Ha mencionado la palabra anti-dogmatismo. ¿Piensa que en la lingüística de los últimos años se ha recaído en posturas dogmáticas?

-Sí. En general, éste es el peligro de todas las disciplinas y más todavía de las disciplinas humanas, porque las ciencias naturales difícilmente pueden ser dogmáticas. En ellas, a lo sumo puede haber dos hipótesis de trabajo y después se trata de estudiar empíricamente las mismas.

Por lo tanto, a lo sumo se mantiene el dogma como hipótesis en los científicos conscientes, con la conciencia de que se trata de hipótesis. Por ejemplo, para estudiar la luz se pone debajo algo para la interpretación, que eso significa hipótesis: suposición. Supongamos que se trate de una transmisión de corpúsculos. Tenemos entonces la teoría corpuscular y se trata de ver si los caminos de la luz se explican de manera satisfactoria así. Se ve que esto no marcha y se pone debajo la teoría ondulatoria. Se dice que la teoría está compuesta por ondas y se ve en qué medida puede servir esto.

En las ciencias humanas no hay en realidad hipótesis con respecto a lo universal, ni puede haberlas porque es absurdo decir que no sabe lo que hace, puesto que el hombre hace él mismo el lenguaje. Enunciar una hipótesis, decir que el lenguaje sea 
esto o aquello es una actitud falsa. Hipótesis se pueden emitir sólo con respecto a lo particular, a lo histórico, pero no con respecto a lo universal. Puesto que aqui la base es siempre el saber intuitivo que tiene el hablante como hablante y, por tanto, el linguista como hablante, entonces este saber es un saber en lo intuitivo absolutamente seguro y al trasladarlo al plano de la reflexividad considera el linguista que podría tener como saber fundado el que tiene como saber intuitivo. $Y$ de aquí entonces el peligro del dogmatismo, porque en ese traslado puede haber habido desviaciones, puede haber habido parcialización. De aquí que sea mucho mayor el peligro de dogmatismo, no ya de tipo externo como podría ser en una ciencia natural el fundado en convicciones religiosas o ideologías políticas, sino dogmatismo con respecto al lenguaje mismo. Aquí es mucho más fácil y esto también sucede en el arte.

Esto se debe a que toda actividad humana tiene su norma o su ética intrínseca, que es la de presentarse necesariamente como el deber ser de esa actividad. Entonces, el que hace lingüística de un modo determinado entiende que así debe hacerse la lingüística, que cualquier otro ser razonable deberia hacerla de ese mismo modo. Ahí puede ser que se equivoque porque tiende a ponerse a sí mismo como norma, con parcializaciones y enfoques erróneos en el sentido que decíamos antes, con relación a una intuición en realidad certera. En la intuición las cosas se dan globalmente y es conocimiento no analizado. Cuando comenzamos a analizarlo, a examinarlo por partes, corremos el riesgo de equivocarnos, de considerar como esencial lo que no es esencial y entonces tratar de imponer este deber ser de nuestra actividad como el deber ser de la actividad de cualquier otro sin preguntarnos si efectivamente nos hemos equivocado, sin tratar de ver cuál puede ser la razón de los otros.

Les pongo dos ejemplos. En la lingüística moderna se ha discutido mucho y se ha rechazado la concepción del significado en Bloomfield. También se ha rechazado el enfoque formal y matemático para todas las unidades lingüísticas que hizo Hjemslev; en este caso se decía que era álgebra del lenguaje sin que se tratara de ver cuáles eran los motivos íntimos de estos dos estudiosos.

No es que Bloomfield no supiera que el lenguaje no significa. Es más, define la forma lingüística siempre como forma que significa y dice, por ejemplo, que toda la gramática es semántica, es decir, que está determinada por el contenido. Lo que sucede es que Bloomfield tiene una determinada concepción de la ciencia. Entiende que la ciencia sólo puede hacerse sobre una base intersubjetiva, en forma behaviorista, estudiando los hechos de conducta exterior, lo que cualquier otro sujeto podría observar o que una máquina bien hecha podria observar. El lingüista tiene que olvidar necesariamente todo aquello que sabe en cuanto hablante, todo aquello que sabe por introspección y dedicarse a aquello que efectivamente puede observar y esto le obliga a considerar que el significado no puede estudiarse. El behaviorismo no admite la introspección, sino sólo hechos de conducta exteriores. Entonces, en lugar de decir que no aceptamos la expulsión del significado, hay que decir -y hay que decir por qué - no aceptamos esta concepción de la ciencia. Porque Bloomfield en realidad es perfectamente coherente con su concepción de la ciencia. Hace un sacrificio enorme y lo sabe. Pero piensa que sólo así puede ser objetivo. En lugar de simplemente decir 
que se equivoca, hay que decir que se equivoca de una manera superior. Se equivoca porque cree que la ciencia tiene que ser así.

El caso de Hjemslev es el contrario. Hjemslev, en vez de una concepción de la ciencia, parte de una concepción del lenguaje por la que entiende que el lenguaje es solamente forma y no sustancia. Ni de la expresión ni del contenido. Que la materia puede ser fónica, escrita o la que sea. En este caso hay que decir que el lenguaje no es así. No se puede decir simplemente que la teoría de Hjemslev esálgebra del lenguaje, sino que hay que decir que se rechaza la concepción misma del lenguaje y decir que no es cierto que el lenguaje sea forma pura, sino que está siempre en forma de una sustancia determinada, y que no es cierto, por ejemplo, que la escritura tenga la misma forma que lo hablado, sino que la escritura es un sistema secundario que puede desarrollarse por si mismo, pero que primero lo que hace es reproducir aquellas unidades que se dan en el lenguaje hablado.

Esto significa precisamente el anti-dogmatismo: entender los motivos de los otros. No decir que se trata de un error, o decir que no se entiende. Decir que algo no se entiende es siempre una expresión de insuficiencia propia; significa en realidad "yo no lo entiendo", "yo no soy capaz de entenderlo".

- A propósito de lo universal, que vd. ya ha mencionado en varias ocasiones, ¿vd. cree que ahora que se ha retomado la idea de una gramática universal, las ideas de Greenberg y Comrie, un lingüista debe presentar una teoría tanto intercomo intralingüística o se debe limitar a la descripción de una lengua sin ir más allá del nivel intralingüístico?

-En esto hay que distinguir entre gramática universal y gramática general. Los universalistas en general hacen gramática general confundiéndola con gramática universal. Esta es un modelo teórico de gramática que puede aplicarse a la descripción de cualquier lengua, mientras que gramática general es una descripción de todas las lenguas en una sola gramática, admitiendo que todas las lenguas son de algún modo iguales y manifiestan las mismas funciones. Esto no puede hacerse porque lo general no es de por sí universal. Se confunde aquí la universalidad esencial o necesaria, aquello por lo cual algo es o no es lo que es. Es o no es lengua en este caso.

Lo que una lengua debe necesariamente presentar es un sistema léxico, un sistema gramatical, los dos planos de la expresión y el contenido, etc., mientras que algo específico es ya general y, de ningún modo, universal necesario. Debemos ver si se da o no en las lenguas. Pero esto siempre significa verlo nada más que en las lenguas conocidas. Si podemos imaginar una lengua sin eso, entonces no es un universal necesario, sino una generalidad histórica en las lenguas conocidas. Cuando Jakobson dice que es un universal del lenguaje el que todas las lenguas tengan silabas abiertas, pero que ninguna tenga sólo sílabas cerradas, esto es una generalización sobre todas las lenguas conocidas. Se puede perfectamente imaginar una lengua que tenga solamente silabas cerradas. Se trata entonces de una generalidad histórica y no de una necesidad racional. Por eso, la teoría de la gramática es siempre gramática universal, incluso la gramática tradicional contiene de algún modo una idea de gramática universal. Por su parte, gramática general significa descripción de todas las lenguas, por lo menos de algunas, como si fueran una sola y esto es enteramente absurdo. Por ello, 
precisamente en las gramáticas llamadas generales, del sigloXVIII, lo que se entiende como teoría gramatical sigue siendo perfectamente válido y lo que, en cambio, pretende ser descripción y atribución de la misma función a todas las lenguas es algo caduco porque no podemos atribuir a todas las lenguas algo que la misma idea de lengua no exija.

Si pensamos en qué debe tener racional y necesariamente una lengua para ser lengua, nada más que dos planos, nada más que los elementos mínimos del plano de la oración. Debe haber algo combinable y una unidad del decir, pero nada más. $\mathrm{Ni}$ siquiera el plano del discurso como plano gramatical es necesario.

En una lengua podría no haber ningún procedimiento concerniente a este plano, sino sólo las oraciones una tras otra. Entonces, no existiría en esta lengua el plano gramatical del texto. Lo cierto es que advertimos que siempre existe. Pero esto es un hecho empírico; no un hecho racionalmente necesario. También es necesario que toda lengua tenga algo para transformar el nombrar en el decir, pero eso se deduce de las funciones generales del lenguaje. Toda lengua tiene que tener algo para nombrar y algo para decir: léxico y gramática, y debe tener algo para la transformación, algo como un verbo.

Cuando Chomsky, en los últimos tiempos trata de deducir los universales del lenguaje, uno se pregunta qué es lo que ha quedado. Parece que $S$ es $P$, nada más que eso. Aunque eso tampoco sería universal, porque una lengua podría no distinguir entre sujeto y predicado, sino entre tema y rema, como el japonés. En japonés, el sufijo - wa se aplica a todo aquello que constituye el tema del hablar. No se dice "Juan viene", sino "hay venir con respecto a Juan". En relación al léxico, lo único que hay que decir es que una lengua debe tener algo para hacer las distinciones necesarias en el ambiente al que corresponde, pero nada más. Las distinciones que se hagan de hecho no son ya necesarias. Las partes, por ejemplo, del cuerpo no tienen que corresponder necesariamente a las que distinguimos. La palabra "mano" podría designar un área corporal distinta. La partición que nosotros hacemos podría ser otra. En latín, manus longa ya no es la mano. Se ha dicho que no podria imaginarse una lengua que tuviera la misma palabra para la mano derecha y para el pie izquierdo. Yo podría imaginar una lengua así, en la que, por ejemplo, la mano derecha y el pie izquierdo se relacionen con algo mágico y que se denominen del mismo modo o se sometan al mismo tabú lingüístico.

Hegel decía que para las cosas concretas, las lenguas tienen distinciones diferentes y daba como ejemplo Sol, Luna, mar. No es cierto porque una lengua podría tener un sólo término para Sol, Luna y estrellas. No es evidente que una acción como "dar" , aunque sea una acción universal, deba tener una unidad léxica para designarla. En japonés hay, por lo menos, cuatro términos diferentes, porque el "dar" puede ser de la primera a la segunda persona o al revés, y de superior a inferior $o$ al contrario. Es labor de la investigación lingüistica ver en qué medida hay en todas las lenguas universales de estos que yo llamo empíricos, de la generalidad histórica.

Lo universal no es algo universal concreto sino la posibilidad de hacer lo mismo en otra parte o en otro campo del sistema lingüístico. Puede haber una lengua con número para los sustantivos y otra con número para los verbos. Como nosotros, que 
en el verbo distribuir, por ejemplo, tenemos la participación de varios destinatarios. Una distinción puede ser algo central en una lengua y marginal en otra. Para comprender las lenguas en tanto diferentes, partimos de esta posibilidad de establecer distinciones análogas en casos distintos. Por ejemplo, llover, nevar no se atribuyen a ningún sujeto en muchas lenguas. En español se puede también decir "se grita", sin atribuirlo a ningún sujeto; o "se come mucho por alli".

La gramática transformativa puede ser válida como teoría de la gramática, pero no cuando intenta describir particularidades de varias lenguas a la vez.

- Ahora parece que se están introduciendo criterios de cientificidad en la lingüística en el sentido de que una gramática es buena si sirve para hacer funcionar una máquina de traducir. ¿Qué opina vd. de esto? ¿No se han cambiado los objetivos del análisis lingüísticos?

- La gramática es una actividad humana y se hace siempre con una determinada finalidad. En cuanto al método - y método significa camino- hay que ver qué método permite la realización de esta finalidad de la manera más sencilla y cómoda, de suerte que entonces no se trata de gramática mejor o peor, sino de gramática mejor para tal finalidad. En el caso de la gramática destinada a la traducción hay que hacer la gramática destinada a esta finalidad.

Los ordenadores trabajan con sistemas binarios y hay que hacer la gramática para que la máquina puede aplicarla. Hay, por ejemplo, que fragmentar las unidades de otro modo.

Dada la finalidad, hay que preguntarse también si la finalidad misma es una finalidad razonable y ésta que ustedes mencionan no es una finalidad razonable. No se puede hacer la gramática de una lengua para traducir textos, porque la lengua no se traduce; se traducen textos y los textos no son sólo lengua. Por tanto, es completamente absurdo hacer una gramática y pretender que sea la mejor. Ninguna gramática va a permitir jamás traducir textos como tales. Eso es imposible puesto que el texto no depende de la lengua sólo.

El hablar es una actividad mucho más compleja y se dan toda la serie de determinaciones relacionadas con el conocimiento del mundo, de los hechos culturales, etc., de suerte que lo que hay que modificar es la finalidad misma y decir que no hay que pretender una traducción de los textos reales, sino una redacción del texto real susceptible de un tratamiento automático. Sería como pedirle a un científico que ha escrito un texto en español que añada un resumen en inglés. En este caso, sería un resumen constituído sólo por lengua y que se pudiese traducir con facilidad

¿Para qué necesitamos la traducción automática? No, por cierto, para traducir a Shakespeare. La máquina no hace más de lo que se le ha dado. Lo hace mucho más rápidamente que el hombre y sin error. Por eso le damos ya un texto construido de este modo. Una máquina puede traducir cifras arábigas a romanas. Muy bien. Pero no se puede uno proponer algo racionalmente imposible

La ventaja de la máquina para el análisis incluso de los textos es que muchas veces nos hace descubrir nuestros propios errores. De ningún modo puede decirse que la gramática sea por esto mejor o peor. La gramática mejor en absoluto, sin ser una gramática para algo, es la que dice las cosas como son. 
En cuanto a la pragmática es poco razonable hacer una gramática de una lengua que sea al mismo tiempo pragmática, porque ésta no concierne a la lengua sino al empleo de la lengua. Quien intenta una concepción integrativa como Dik, al llegar a hacer distinciones en la pragmática sólo establece dos funciones

-Sí, tópico y foco.

- Si, efectivamente es reducir la pragmática a casi nada. Lo que es una confesión de la imposibilidad de integrarla. Por otra parte, desde el punto vista de la teoría no hay objeto propio de la pragmática. La pragmática es una parte de la lingüística del texto

- ¿No debe considerarse dentro de la teoría como un componente más al lado del sintáctico, etc.?

No, porque las lenguas son sistemas léxicos y gramaticales pero no pragmáticos. Las funciones pragmáticas son siempre textuales, del hablar, y estas funciones entonces podrian estar organizadas de modo que habría que hacer para cada lengua una gramática del empleo, pero esto significa no sólo con respecto a lo que se llama pragmática, que nunca está muy bien definida, sino con respecto a todos los factores del hablar: lo que se habla, a quién, dónde se habla, etc.

En general, yo soy contrario por razones muy particulares a la noción misma de pragmática, porque se trata de uno de estos engendros del positivismo que distingue las tres disciplinas por las relaciones del signo: el signo y las cosas designadas, el signo con otros signos y el signo con el usuario.

Esto no es aplicable a las lenguas llamadas naturales, que son las únicas que existen. Las otras no son lenguas, sino códigos. Esto es una falsificación de las lenguas. Las lenguas no tienen signos que se relacionen con signos_significantes con significantes_, sino siempre contenidos que se relacionan con contenidos y no se relacionan los signos con las cosas, sino los significantes con significados dentro de la lengua misma. La cosa, lo designado, queda fuera. Lo designado es lo no semántico. La lengua tiene significado para virtualidades. En ese sentido, los problemas son problemas reales, pero colocarlos en una disciplina y pensar que esa disciplina está coordinada con la sintaxis y la semántica, eso no es nada cierto.

-Sobre esta misma pregunta ; vd. cree que los objetivos tradicionales no han sido relegados a pesar de que haya otros enfoques?

- Yo creo que otros enfoques no pueden ni siquiera emprenderse sin el conocimiento de las estructuras de las lenguas, porque siempre se tratará del empleo de tal o cuál estructura. Se establecen ahí las determinaciones ulteriores de una estructura para determinadas finalidades o situaciones. Lo que constituye aquí error es creer que un enfoque nuevo reemplaza al enfoque, digamos, puramente descriptivo del sistema. No lo reemplaza, sino que es aditivo. Plantea otro problema nuevo. Ninguna disciplina puede resolver problemas que no plantee. ¿Cómo puede resolver la pragmática el problema de la estructura del significado en una lengua, si no plantea este problema?

Les pondré un ejemplo. Cuando se ha hecho la estética empírica, ni siquiera se plantea el problema de qué es el arte. En consecuencia, no se puede resolver. Lo que no quiere decir que la estética empírica carezca de interés, pero esta disciplina plantea otros problemas; no el de qué es el arte. Aquí hay que advertir la necesidad de 
disciplinas que nos expliquen no sólo la estructura de la lengua, sino también su empleo. A esto vienen a contribuir la pragmática y la lingüistica del texto en sus diversas formas. Se advierte que la descripción de una lengua todavía no nos dice nada sobre el uso. Cuando tenemos que aplicar la descripción a la enseñanza o a la traducción, la lengua nos dice bien poco. Nos dice cuáles son los instrumentos, pero no cómo se emplean. Hace falta la disciplina del empleo que yo llamo en alemán Linguistik der Sprachverwendung, o sea, lingüistica del uso de la lengua. En la lingüística tradicional, esto se hacía indirectamente. Primerose describían las cosas y después se decían para qué servian las construcciones. En la sintaxis latina se decía que la finalidad, por ejemplo, se expresaba de varias maneras como legati venerunt ad pacem petendam, o pacem petituri, o pacem petentes, o pacis petendae causa, etc. O sea, decía algo del empleo, aunque no mucho. En la descripción estructural todo esto se deja de lado. Si, por ejemplo, dentro de la fonética nace la fonología, entonces la fonología se delimita con respecto a la fonética y entonces tenemos algo muy bien hecho como sistema de la lengua, pero que no nos dice cómo hay que pronunciar. Esto sucede en todas las disciplinas en que se delimite algo con respecto a lo real, aunque concierna a cosas absolutamente esenciales. Las unidades que disciplinas como la fonología distinguen no constituyen el empleo real, el cual no puede aclararse totalmente porque es infinito, pero hay tipos. Entonces una lingüística del empleo de la lengua no nos explicará todo lo que podamos encontrar en Joyce, pero sí los tipos y eso nos permitirá también entender lo que esta gramática no ha previsto.

- Pasemos, si le parece, a temas relacionados con su semántica. La distinción entre significado lingüístico y designación separa su teoría de muchas otras. Sin embargo, parece que ha de quedar muy poco terreno para el significado estructurado lingüísticamente. Hay también otra cuestión, relacionada con la que algunos autores estudian bajo la distinción de definiciones de diccionario y definiciones de enciclopedia. Nos referimos a que vd. habla en la "Introducción al estudio estructural del léxico" de la escasa distancia que separa las clasificaciones populares de las científicas.

- Lo primero que tiene hacer la semántica de una lengua para establecer su estructura es la distinción entre léxico estructurado en tal lengua determinada y el léxico no estructurado, que puede ser tanto de ciencia, como de ciencia y técnica popular. La botánica no deja de ser botánica por ser botánica popular, pero no pertenecen al léxico estructurado todos los nombres de las plantas, ni todos los nombres de los peces, porque la lengua no distingue esos nombres mediante una distinción de rasgos distintivos idiomáticos. A lo sumo, puede, como distingue el español, distinguir en el caso de los peces entre pez y pescado. No están esos nombres ordenados por la lengua, sino que constituyen una nomenclatura abierta. Es totalmente inútil tratar de buscar definiciones idiomáticas en español o en la lengua que sea.

¿Qué es lo que hay que decir? Pues lo que siempre se ha dicho: que en este caso se trata de describir los objetos, de dar una imagen del objeto. No se puede pretender que, por ejemplo, pertenezcan al español todos los nombres de peces, todos los nombres de partes del arado, de partes del zapato o los cientos de miles de términos de la química. En estos términos el significado coincide con la designación. Sólo se 
puede describir el objeto como tal y no se puede intentar distinguirlo mediante rasgos semánticos de la lengua si la lengua no lo hace. El principio es el mismo que habíamos enunciado antes: decir las cosas como son, es decir, si la lengua no hace ahí distinción mediante rasgos distintivos, entonces no cabe buscar rasgos distintivos.

El español distingue entre llevary traer mediante rasgos idiomáticos del español, pero no hay que incurrir en la confusión ni en uno ni en otro sentido; no cabe decir que todo lo que la lengua designa es asunto de ciencia, pero tampoco lo contrario. Cabe decir que hay una gran parte del léxico donde lo propio de la lengua es sólo el significante. Lo designado podría ser exactamente lo mismo en muchísimas lenguas. Nosotros preguntamos cómo se llama la rosada en francés y no la oponemos a mero. Ahí no hay oposición, sino tal cosa o tal otra.

-Vd. en el antes citado ensayo "Introducción al estudio estructural del léxico", dice que "el conocimiento de las cosas y las apreciaciones y opiniones concernientes a las cosas son importantes en lo que se refiere a la fraseología metafórica." ¿Este tipo de asociaciones entre las cosas es algo que cabría estudiar con los métodos de la semántica?

-Sí, pero hay que advertir que no se trata de algo idiomático. La lengua es algo muy complejo porque la lengua es mediadora, es decir, a través de ella nombramos las cosas y entonces pensamos que ciertas asociaciones lo son con la palabra, mientras que son con la cosa nombrada. Por ejemplo, decía Ortega en "Miseria y esplendor de la traducción" que jamás se podrá traducir al español la palabra Wald por la imposiblidad de traducir todos los sentimientos que pueda despertar en el alemán la palabra Wald ; pero esto no es cierto, porque esos sentimientos no son con respecto a la palabra sino con respecto al bosque mismo. El alemán tiene cierta relación con el bosque, pero esto no puede traducirse porque no está expresado. Puede traducirse sólo aquello que constituye relación semiótica del lenguaje; lo otro no lo puede traducir. Eso otro, el traductor lo puede explicar o añadir.

Es idiomática la distinción dentro de una lengua determinada dentro de un campo semántico dado. Por ejemplo, en latín bos corresponde a bueyo vaca, vacuno o bovino, en español. Vaca es algo dentro del término bos ; para el español, ya no. La relación de buey con arado, yugo, labranza y las otras cosas que menciona Charles Bally es una relación del buey mismo. Al encontrar buey en un texto, designa la cosa. Como el léxico es lo más cercano a la cosa - la gramática ya no está tan cerca-, pasamos a la realidad de la cosa. La palabra buey nombra al buey y a través de éste sugiere labranza, etc. Porque el buey se conoce en este contexto en nuestras comunidades, pero en las comunidades donde se conozca el buey en relación con el templo, con lo sagrado, etc., las asociaciones serán muy diferentes. Y estas cosas no se dan en francés como dice Bally, sino en todas las comunidades donde el buey se da como animal de labranza. Entonces, lo que hay que decir, cuando se describe la lengua, es que estas asociaciones son no lingüísticas. Cuando se trata de establecer el sentido del texto, hay que decir que estas asociaciones funcionan también en el texto, porque éste no está constituído sólo por lengua, sino también por todo este transfondo. Si alguien le dice a otra persona "Esto es un buey", como valor de texto podrá tener sentidos totalmente diferentes en comunidades distintas. Cuando se traduce de una 
comunidad a otra muy diferente, muchas veces hay que aclarar y decir el sentido de una expresión en la primera comunidad.

Un buen ejemplo son los valores simbólicos de los colores. En Oriente, por ejemplo, los puntos cardinales han llevado asociados colores. Negro, por ejemplo, significa septentrional. Entonces, el Mar Negro es el mar que se encuentra al Norte. Para nosotros, el negro es símbolo de duelo, tristeza, etc. y el blanco de alegría o, cuando menos, de inocencia, serenidad, etc. Para los japoneses es al revés: El blanco es el color de la tristeza, de la soledad, etc. El negro, de la alegría. En la película japonesa La puerta del infierno, todo lo que se ve durante los primeros minutos es blanco. Esto expresaba una gran tristeza para los japoneses; mientras que para nosotros, en absoluto.

Entonces, con esto surgen expresiones que han de explicarse no mediante la palabra, sino, como en este caso, con el color mismo. El lenguaje es un continuo desde las cosas designadas, "el mundo", hasta la estructura total del texto, y ésta es la parte más alejada de la realidad. Al revés, lo más cercano a la realidad es el léxico. El léxico está ahí, recubriendo y organizando el mundo. Es muy fácil confundir la palabra con la cosa. Esto incluso puede verse en el sentido mágico de las palabras, por ejemplo cuando hay que nombrar.

- Usted afirma en varios lugares la acción creadora del lenguaje. ¿Qué podría decirnos sobre esto, que manifiesta una posición a la que se oponen los intentos de automatizar el lenguaje?

- Será mejor que lean lo que digo en El hombre y su lenguaje, en las "Cinco tesis sobre lenguaje y poesía". Este tema es muy largo de explicar y les remito a los textos. Es necesario explicar qué se entiende por la identidad de lenguaje y poesía. No se afirma que toda frase dicha es poesía. Jamás nadie ha pensado en esto. Croce no era ningún estúpido. No pensaba que "Deme vd. un vaso de agua" constituya poesía. Lo que se entiende es que el lenguaje como actividad creadora y la poesía son actividades de la misma índole. El lenguaje es lo universal dentro de lo individual, o sea, un modo de ser universal dentro de la unidad de incluso una sola palabra.

Como la poesía, el lenguaje es anterior a la distinción entre existencia e inexistencia, verdad y falsedad. En la poesía, si se habla de una guerra tal o cual, eso no constituye un documento, no significa que haya tenido lugar. Del mismo modo, en el lenguaje la existencia de una palabra no implica existencia o inexistencia de la cosa. Aristóteles llega tan lejos que dice que no sólo las palabras de las que sabemos que no implican existencia como $\tau \rho \alpha \gamma \varepsilon \lambda \alpha \phi \circ \varsigma$, sino que ni siquiera la palabra $\alpha \nu \Theta \rho \omega \pi \circ \varsigma$ implica que el hombre exista. La palabra es nada más que un modo de ser, pero absolutamente virtual y si corresponde a algo o no, es algo que debemos comprobar. Es también anterior a la verdad o a la falsedad.

Fíjense también en que para comprobar la existencia es necesario tener ya el lenguaje, o sea, saber qué se va a buscar, porque la existencia no es una propiedad, sino una relación entre un objeto mental y un objeto que encontramos fuera de la conciencia. Para comprobar si los centauros existen, primero tenemos que saber qué significa centauro. Pero si la existencia puede comprobarse, la inexistencia absoluta no puede comprobarse jamás. Sólo diremos que algo no existe si es en sí mismo contradictorio. 
Y lo mismo con respecto a la verdad. La verdad es la relación entre lo dicho y lo que hay. Para establecer si algo es verdadero o falso se necesita el lenguaje. El lenguaje como tal es anterior. Platón dice precisamente esto, que juicios como "Teeteto vuela" o "Teeteto está sentado" no son de por sí ni verdaderos ni falsos, y sólo lo serán si se aplican a Teeteto que está sentado o que no está sentado.

Es importante lo que se quiere decir con la identidad entre lenguaje y poesía: que como actos de creación son actos del mismo tipo. 